\title{
INTEGRATION OF VIDEO AND RADIATION ANALYSIS DATA
}

\author{
H. O. Menlove, J. A. Howell, C. A. Rodriguez, G. W. Eccleston, D. Beddingfield, J. E. Smith \\ Los Alamos National Laboratory \\ Los Alamos, NM 87545 USA) \\ C. W. Baumgart \\ EG\&G Energy Measurements, Inc. \\ Los Alamos, NM, USA
}

\begin{abstract}
We have introduced a new method to integrate spatial (digital video) with time (radiation monitoring) information. This technology is based on pattern recognition by neural networks, provides significant capability to analyze complex data, and has the ability to learn and adapt to changing situations. This technique has the potential of significantly reducing the frequency of inspection visits to key facilities without a loss of safeguards effectiveness.
\end{abstract}

\section{INTRODUCTION}

For the past several years, the integration of containment and surveillance $(C / S)$ with nondestructive assay (NDA) sensors for monitoring the movement of nuclear material has focused on the hardware and communications protocols in the transmission network. Little progress has been made in methods to utilize the combined $\mathrm{C} / \mathrm{S}$ and NDA data for safeguards and to reduce the inspector time spent in nuclear facilities. One of the fundamental problems in the integration of the combined data is that the two methods operate in different dimensions. The $\mathrm{C} / \mathrm{S}$ video data is spatial in nature; whereas, the NDA sensors provide radiation levels versus time data.

We have developed a new method [1] to facilitate this integration, called Video Time and Radiation Analysis Program (VTRAP). Since the interplay between the multiple regions of interest in the video motion data and the multiple radiation sensors as a function of time is very complex, we have introduced neural network analyses to evaluate the data and distinguish abnormal events from normal activities. This approach is well suited for large automated facilities where the movement of nuclear material is restricted by the constraints in the robotics system. It would also find applications at reactors, spent fuel storage facilities, reprocessing plants, and nuclear material storage vaults.

\section{VIDEO METHOD}

Traditional $\mathrm{C} / \mathrm{S}$ systems use video simply as a recording mechanism that may be either time lapsed or externally triggered. In the VTRAP we have developed an intelligent video processing component [2,3] that continuously monitors the area of surveillance for changes such as human motion or the movement of robots, containers, etc. Changes in the scene are quantified using a metric, providing motion levels versus time in addition to the spatially oriented video data. In this 


\section{DISCLAIMER}

Portions of this document may be illegible in electronic image products. Images are produced from the best available original document. 
system the video component becomes another detection element as well as a traditional recording instrument.

For example, video detection data representing human entry to a process or storage area is shown in Fig. 1. Once quantified, the data can be represented in numeric form and is easily processed along with other sensor data collected by VTRAP. The spatial video data can then be collected, compressed, and stored, providing a video record of the event if desired.

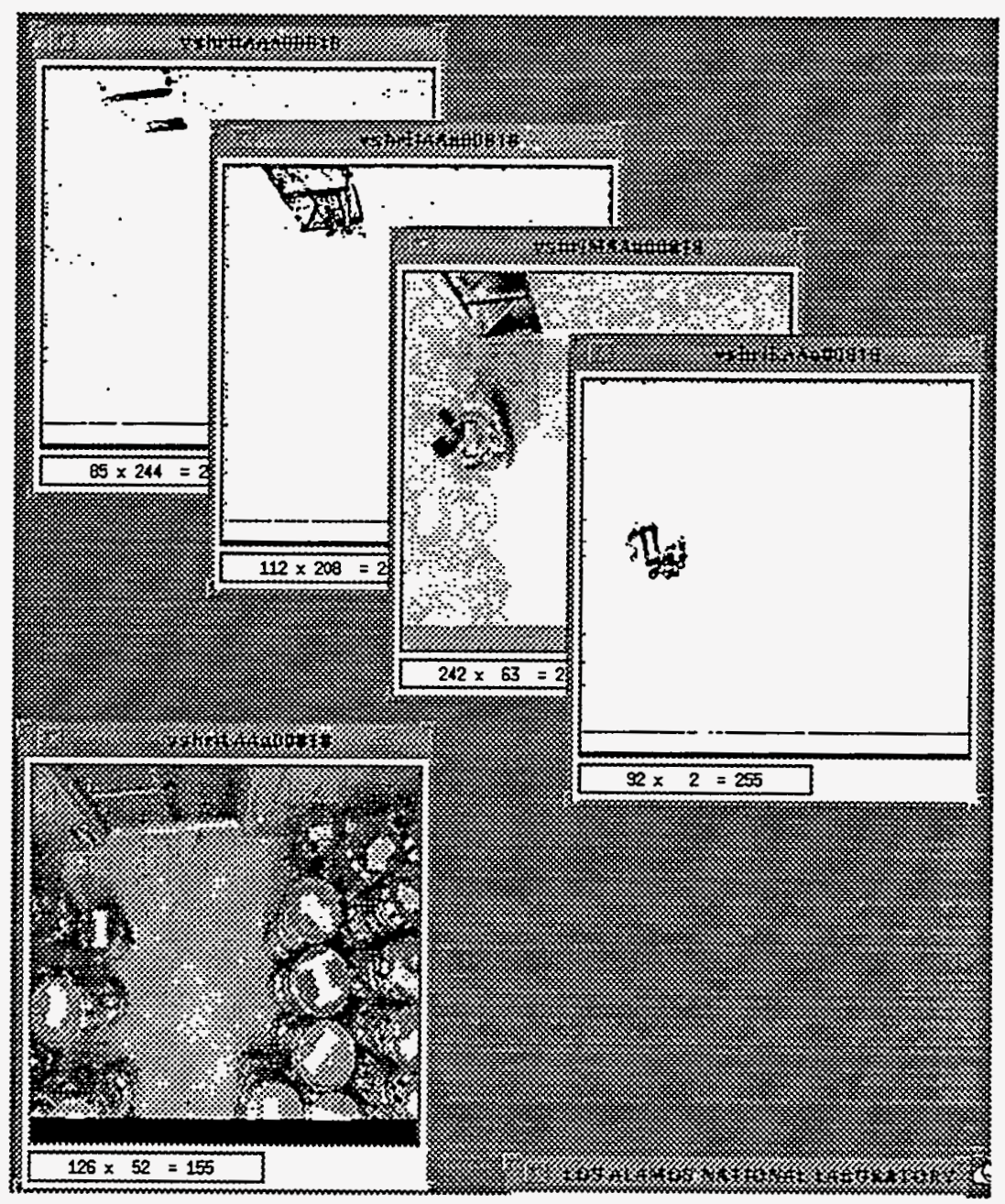

Fig. 1. Video detection data (top, left to right) detecting access to a vault and subsequent removal of an item

In addition to the continuous video detection and video recording methods described above, VTRAP provides a "Region of Interest" feature that allows one or more area(s) of surveillance to be defined. These areas can then be considered independently of each other or in relation to each other when the video detection data is processed with data from other sensors. 


\section{RADIATION SENSORS}

Sensors for a VTRAP system can include radiation detectors such as neutron counters, gamma-ray detectors, and infrared detectors as well as electromechanical sensors [4]. When the monitoring target is special nuclear material such as plutonium, irradiated fuel, or nuclear weapons components, the penetrating radiation signals are the primary signals that get integrated with the video motion signals.

A typical neutron sensor is shown in Fig. 2 including a ${ }^{3} \mathrm{He}$ tube in a polyethylene moderator, a PDT-110A preamplifier, the portable shift register (PSR) electronics [5], and a LON module for connection to the data collection network. The PSR unit supplies the $h v$ bias, preamplifier power, data collection, coincidence logic, and data transmission to the collect computer.

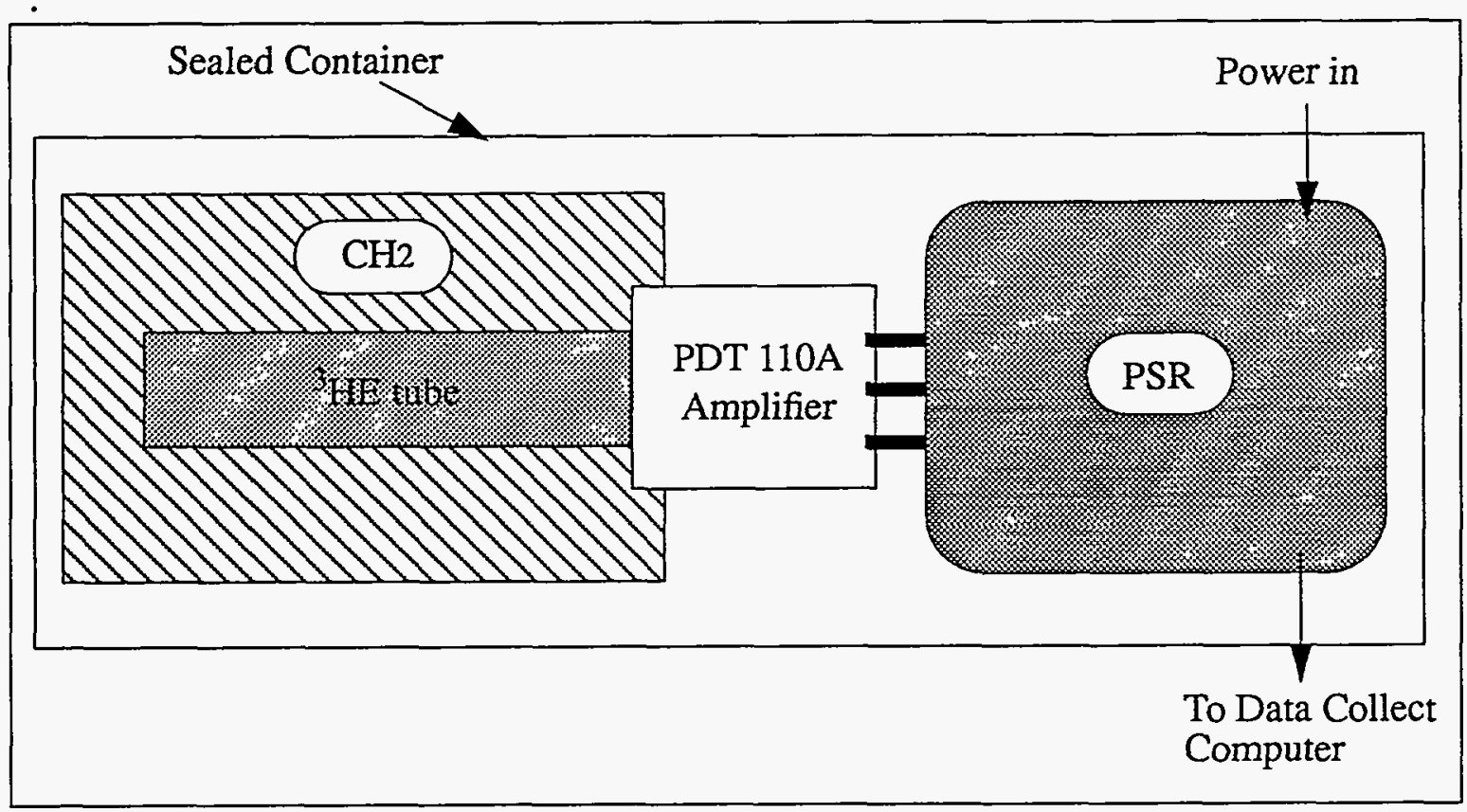

Fig. 2. Schematic diagram of a neutron radiation sensor including the ${ }^{3} \mathrm{He}$ detector tube, a PDT-110A amplifier, and the Portable Shift Register (PSR coincidence electronics. The PSR has local data storage and ten hour battery backup for power outages.

\section{EXPERIMENTAL VTRAP TEST BED}

To develop and evaluate the VTRAP concept, we have established a test bed in a controlled radiation laboratory at the Los Alamos National Laboratory. The equipment includes a nuclear material vault, a radioactive source shield, a neutron slab totals detector, a neutron coincidence detector (HLNC-II), and a digital video camera. The location of the equipment is shown in Fig. 3. 


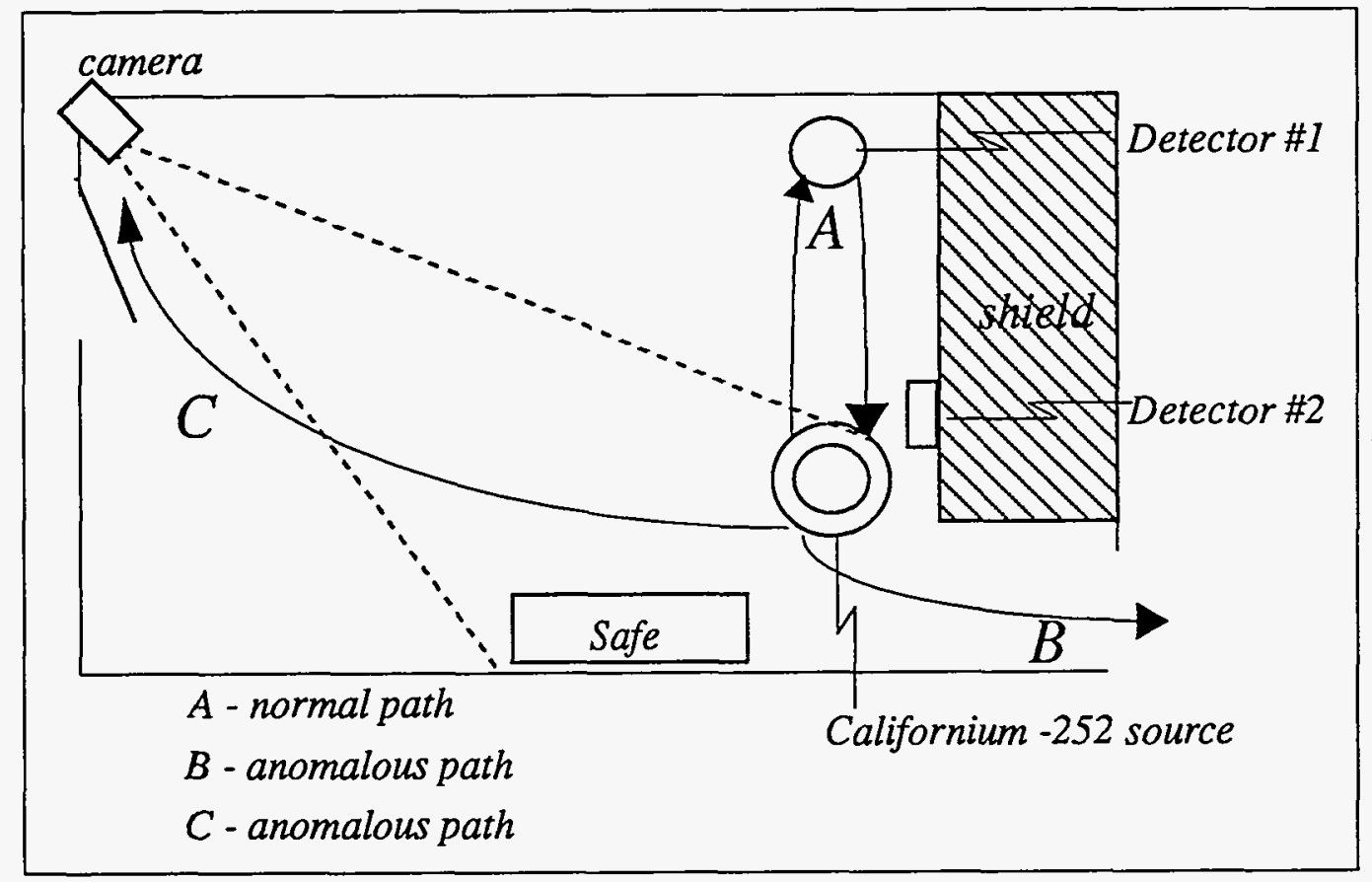

Fig. 3. Diagram of the radiation laboratory for testing the VTRAP sensors including the video camera, a neutron totals counter, a neutron coincidence counter, and a shield or vault for storing radioactive sources. The movement pattern A corresponds to normal neutron source movement; whereas, $B$ and $C$ represent abnormal movements for diversion scenarios.

Experimental procedures were defined to collect a database for "normal" activity to train the neural network analysis. This normal activity included the movement of a small radiation source $\left({ }^{252} \mathrm{Cf}\right)$ from the neutron shield, past the slab detector on the wall, and into the coincidence counter for about a 1-min. count. The source movement was then reversed back into the shield. This movement pattern for the multiple sensors is shown in Fig. 3 where first the video motion detector picks up the person in the vault area. After logging in the SNM movement, the source is raised past the slab detector resulting in the totals rate peak. The person then leaves the video beam (see Fig. 3) and enters the source into the coincidence counter giving both the totals and coincidence counts shown in the bottom two frames of Fig. 4. For the return of the source to the storage area, we see a second peak in the slab detector and the video motion detector. In this work area, it is normal to have a variety of physical motions such as people walking through the area. These physical motion spikes (shown in Fig. 4) are considered "normal" for training the neural network. For this work area, the video motion events are an order of magnitude more numerous than the significant events involving nuclear material movement.

\section{DISCLAIMER}

This report was prepared as an account of work sponsored by an agency of the United States Government. Neither the United States Government nor any agency thereof, nor any of their employees, makes any warranty, express or implied, or assumes any legal liability or responsibility for the accuracy, completeness, or usefulness of any information, apparatus, product, or process disclosed, or represents that its use would not infringe privately owned rights. Reference herein to any specific commercial product, process, or service by trade name, trademark, manufacturer, or otherwise does not necessarily constitute or imply its endorsement, recommendation, or favoring by the United States Government or any agency thereof. The views and opinions of authors expressed herein do not necessarily state or reflect those of the United States Government or any agency thereof. 


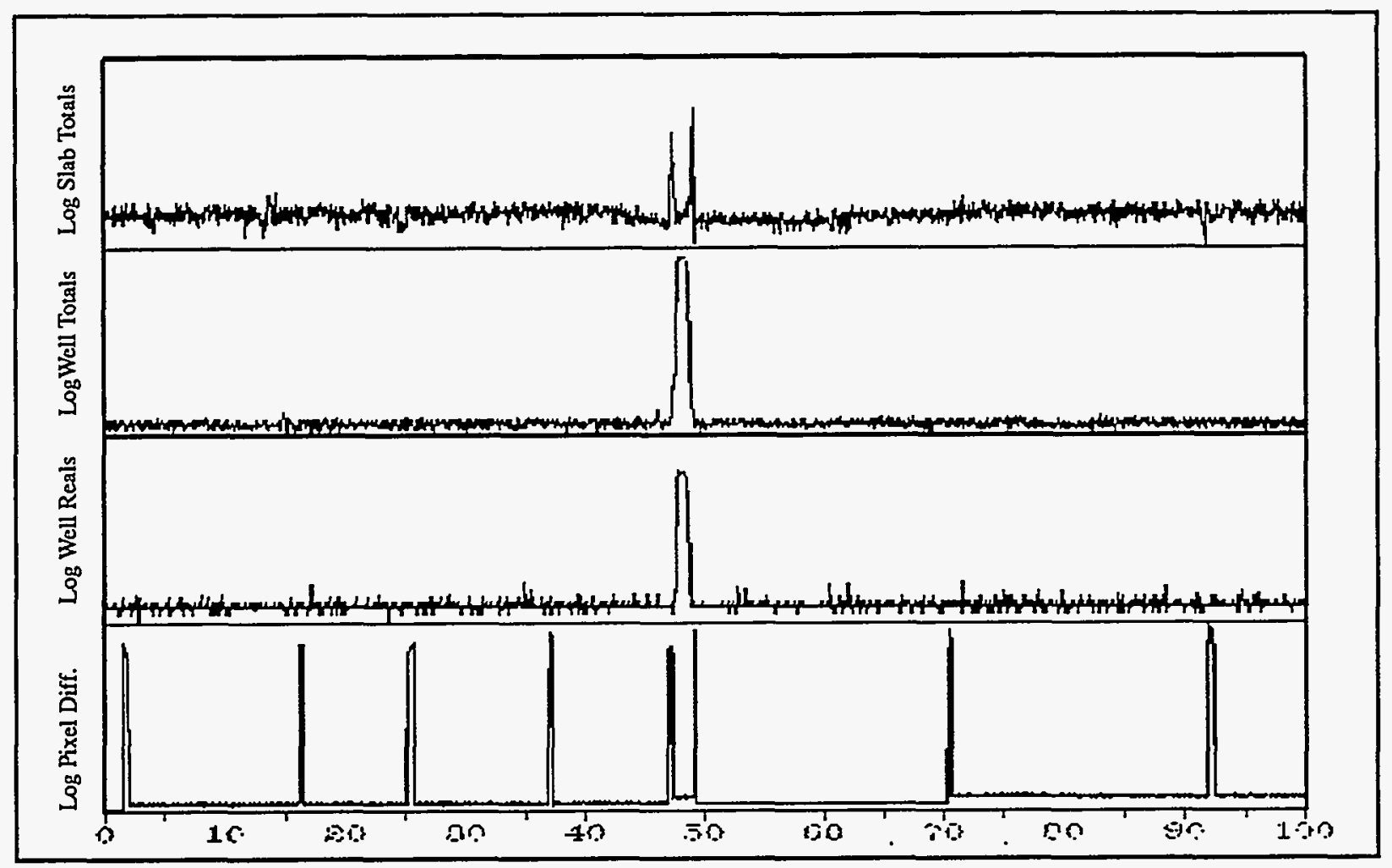

Fig. 4. Normal activity (A in Fig. 3) time responses for the video pixel difference metrics and the radiation sensors including the neutron slab neutron detector, the HLNC-II totals rate, and the HLNC-II coincidence rate.

Figure 5 shows a time enlargement of the significant event shown in Fig. 4 . We see the distinctive pattern related to a normal event. The event patterns contain significant information in magnitude, shape, and time coupling between sensors with the proper arrangement of sensors; it should be possible to determine the radiation source location by means of triangulation. 


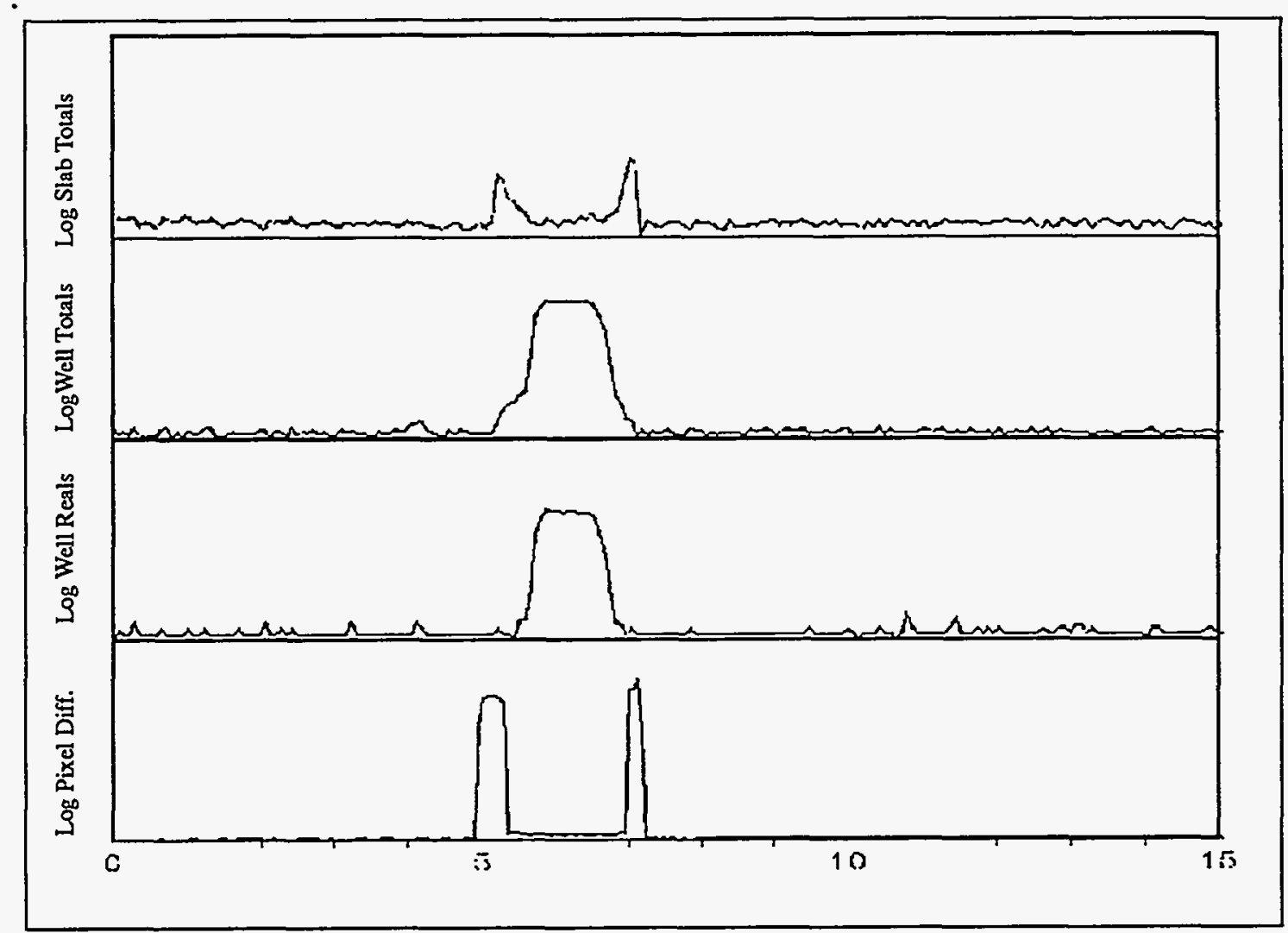

Fig. 5. A time expansion of the radiation event in Fig. 4 where the top pixel difference curve shows the SNM custodian entering and leaving the region of interest twice to remove the source from the shield and then to return it after the assay in the HLNC-II. The two neutron radiation sensors show the pattern for the normal source movement in the lower frames of the figure.

After collecting many ( -100$)$ normal event patterns, a training sequence for abnormal events was initiated. The first abnormal event was for a person to enter the room, remove the source from the shield, and exit through the back door carrying the source. The second abnormal event was to remove the source from the shield and walk past both detectors on the way to the front exit. Figure 6 shows the patterns associated with the abnormal events. 


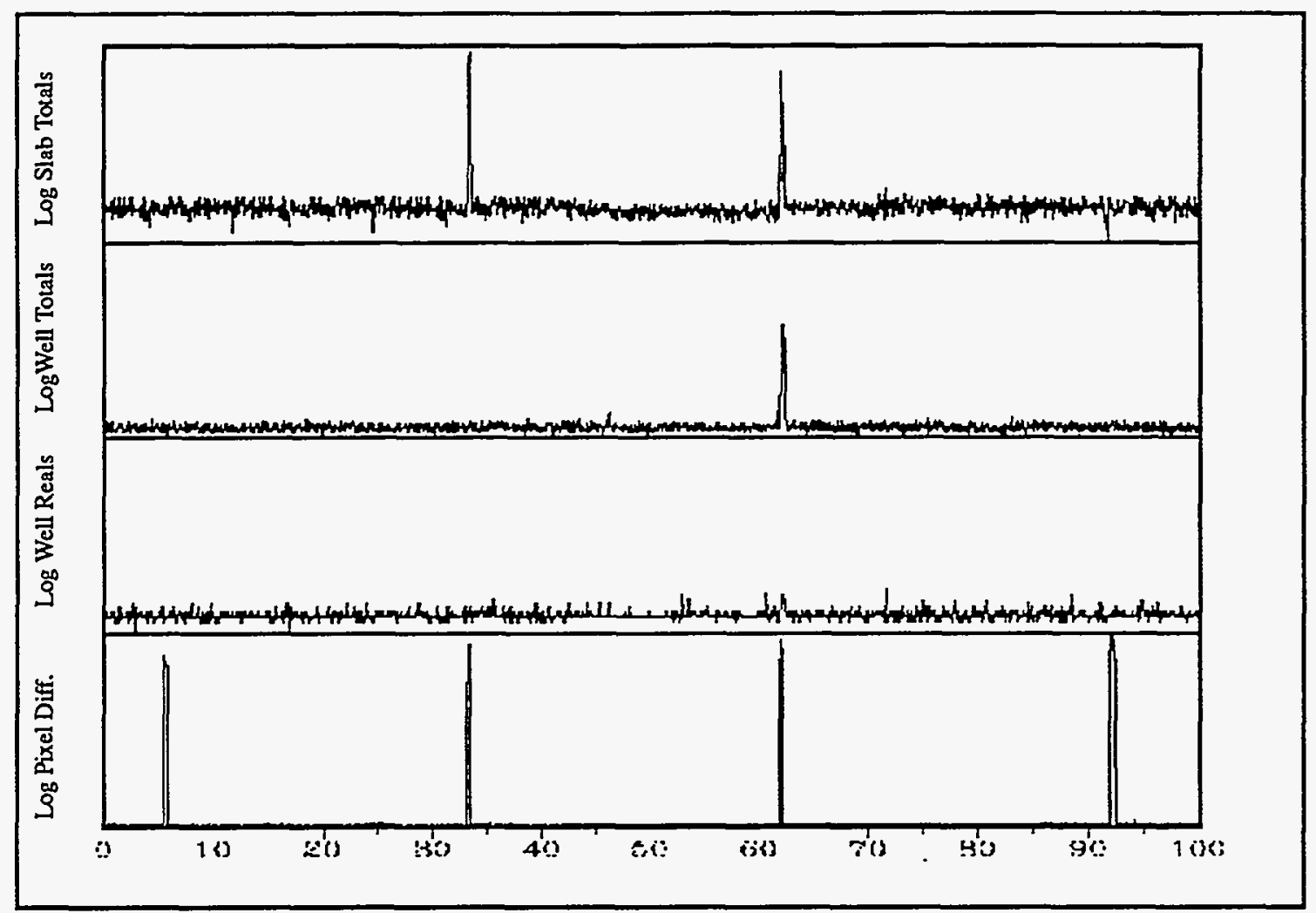

Fig. 6. The time response from the multiple sensors for abnormal source movement representing diversion scenarios where the neutron source is removed from the shield and taken from the room through the back door (pattern $B$ ) or the front door (pattern $C$ ).

The normal and abnormal data sets were supplied for the pattern recognition software in the neural network analysis. The data time steps were $3 \mathrm{~s}$ and all of the sensors were time synchronized before the pattern evaluation. In practice, the time frequency for data collection should be matched to the shortest diversion scenario so that sufficient data are taken to identify the patterns associated with the event.

\section{NEURAL NETWORK APPLICATION}

A neural network is an iterative numerical technique that facilitates the solution of a number of different types of problems including pattern recognition and categorization of data. A neural network learns to recognize patterns by repeatedly examining examples of those patterns [6]. For example, a network could be trained to learn to recognize radiation signatures and video images. Neural networks are named because they are similar to biological neurons and their connections. They have attracted attention recently, and now that hardware implementations are available, they have greater appeal for data acquisition and control systems. We have demonstrated the usefulness of neural network pattern recognition for a variety of safeguards applications [7].

The basic pieces of a neural network are the layers (usually three or more), consisting of several neurons or nodes connected together with adjustable weights (see Fig. 7). The weights are selected such that for a given pattern of input values, a particular output value will be chosen. The most commonly used neural network paradigm is the back propagation algorithm. The network is 
developed through a training step and a testing step. During the training step, examples of input vectors are presented to the network together with a desired output. The actual output is computed and compared with the desired result. Any difference between the two is a measure of the error in the chosen weights. This error is then "back propagated" through the network (fed through the layers in reverse order) and used to update the weights. This type of learning is called "supervised" because the desired-result value controls and directs the process. Learning without such a control is called "unsupervised."

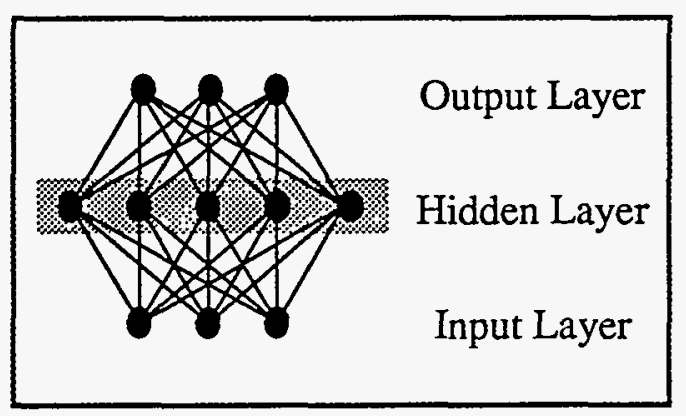

Fig. 7. Neural network architecture

During the testing step, new vectors that have not been used for training are presented to the network. The accuracy of classification of these new testing-phase vectors provides a measure of how well the network was trained.

The interplay between the video motion data and the multiple radiation sensors as a function of time becomes very complex. We have introduced neural network analyses to evaluate the data to distinguish abnormal events from normal activities. Thus, the network becomes a pattern recognizer, alerting the user when an anomalous event has occurred. In a production environment, the neural network methods can be automated to handle the large quantities of data that are generated with frequent collection periods $(1-5 \mathrm{~s})$.

Advantages to this system are as follows:

- adaptability (it learns patterns of activity and can adapt to changing situations),

- efficiency in reviewing and correlating large quantities of information that might otherwise have to be checked manually by an inspector,

- availability of hardware implementations that will make integration with a data acquisition and control system more efficient.

\section{CONTINUITY OF INFORMATION}

To effectively draw safeguards conclusions from sensor data, it is not only necessary to know when significant events happen but it is also highly desirable to have the actual data for the time continuum between the major events. For radiation sensors and our pixel difference metric, we collect a continuum of data from each time step (1-3s). This time-synchronized data is condensed and stored in our collect database. 
When performing a data review, the user can display the time data in a graphical display that allows the historical information from the multiple sensors to be rapidly compared. Questions as to missing triggers in front-end triggering systems are nonexistent with the VTRAP system.

A common problem in systems that are triggered by sensor spikes is the threshold setting for the magnitude of a significant event. As the events get smaller, the threshold tends to get buried in the noise that consists of both random and systematic fluctuations. The data analysis in the VTRAP system can be more sensitive to "significant events" because the continuum data can be analyzed with the combination of the multiple sensors to identify systematic fluctuations that are linked between sensors.

As a simple example of this sensor coupling to reduce systematic fluctuations, consider the pixel difference metric from several different spatial regions of interest. A common background change (systematic) in the pixel difference value comes from changes in the room lighting. By setting one of the regions of interest on a location that is light sensitive but void of normal motion (e.g., the ceiling), the analysis can identify and remove this problem.

Once a significant event has been identified by the neural network analysis, it is often desirable to revisit the continuum data before and/or after the event with a more fine-toothed comb to aid in the interpretation of the event. The continuum database in a VTRAP system makes this possible, whereas, for trigger-type systems, this is not possible.

Because of the large amount of data involved, we take full advantage of commercial data compaction techniques as well as automated data review using neural network pattern recognition methods.

The continuity of knowledge that is inherent in the VTRAP concept is one of the most important advantages of this new technology.

\section{RESULTS AND CONCLUSIONS}

We have developed software to automatically review and analyze safeguards data from continuous unattended monitoring systems. This technology, based on pattern recognition by neural networks, provides significant capability to analyze complex data and has the ability to learn and adapt to changing situations. The VTRAP concept is a new method to integrate spatial (digital video) with time (radiation monitoring) information. Using this technique, we are able to distinguish normal material movements from abnormal activity.

This method involves transforming a video image to a single pixel difference based on the amount of change between video frames and allows a comparison in time with the radiation sensors that are synchronized with the video motion data. The compression of the data is enhanced by the interplay between the synchronized video and NDA data, especially in facilities with activity that is unrelated to nuclear material movement. The essentially continuous time information provided by the VTRAP approach using multiple and redundant sensors gives the potential of significantly reducing the frequency of inspection visits to key facilities without a loss of safeguards effectiveness. 


\section{REFERENCES}

1. J. A. HOWELL, H. O. MENLOVE, G. W. ECCLESTON, R. WHITESON, C. A. RODRIGUEZ, J. K. HALBIG, S. F. KLOSTERBUER, and M. F. MULLEN, "Safeguards Applications of Pattern Recognition and Neural Networks," LA-UR-94-169, presented at IAEA Symposium on International Safeguards, Vienna, March 14-18, 1994.

2. C. RODRIGUEZ, "Video Image Processing for Nuclear Safeguards: The Experimental Inventory Verification System," Los Alamos National Laboratory Technical Report N4/93343.

3. J. N. BRADLEY, C. M. BRISLAWN, J. E. BROWN, C. A. RODRIGUEZ, L. A. STOLTZ, "Video Image Processing for Nuclear Safeguards," Los Alamos National Laboratory, Los Alamos, NM 87545, Presented at the IEEE Conference on Image Compression, Snowbird Utah, April 93.

4. J. K. HALBIG, S. F. KLOSTERBUER, R. H. AUGUSTON, G. E. BOSLER, H. O. MENLOVE, and M. C. MILLER, "Applications for Unattended, Remote NDA Instrumentation," in Conference Record of the 1991 IEEE Nuclear Science Symposium, Catalog \#91CH3100-5, Vol. II, (1991) pp. 1246-1253.

5. J. E. SWANSEN, N. ENSSLIN, M. S. KRICK, AND H. O. MENLOVE, "A New Shift Register for High Count Rate Coincidence Applications," Los Alamos National Laboratory report LA-6788-PR (1977) pp. 4-6.

6. D. E. RUMELHART, and J. L. MCCLELLAND, "Parallel Distributed Processing: Explorations in the Microstructure of Cognition," The MIT Press, Cambridge, Massachusetts (1986).

7. J. A. HOWELL, "The role of neural networks in safeguards and security," J. Nucl. Mater. Manage. 204 (1993) 33-40. 